\title{
Continuous-wave mode-locked visible upconversion laser
}

\author{
P. Xie and S. C. Rand \\ Division of Applied Physics, University of Michigan, 1049 Randall Laboratory, Ann Arbor, Michigan 48109-1120 \\ Received April 27, 1992 \\ We describe continuous-wave mode locking and $Q$ switching of an $\mathrm{Er}: \mathrm{LiYF}_{4}$ trio upconversion laser operating in \\ the green spectral region.
}

A continuing challenge in ultrafast technology is the need for pulsed optical sources at short wavelengths for spectroscopy, communications, and metrology. There are currently no continuously mode-locked laser sources available at wavelengths shorter than that of the argon-ion laser at $458 \mathrm{~nm}$. Cw modelocked dye lasers operate only to $\sim 500 \mathrm{~nm},{ }^{1}$ and solid-state sources exist only at wavelengths longer than approximately $700 \mathrm{~nm}^{2}$ Considerable research effort has therefore been directed to extending operation of short-pulse infrared sources such as the Ti:sapphire laser to shorter wavelengths through nonlinear harmonic generation. ${ }^{3}$ Also, wide-gap semiconductor lasers are under development. ${ }^{4}$ However, there have been no reports of mode locking of short-wavelength solid-state lasers. Despite many desirable properties, solid-state lasers typically operate at photon energies far below band gap and suffer deleterious color-center formation when short-wavelength optical excitation is used. It is no coincidence that even conventional optically pumped, cw solid-state lasers have not been demonstrated at short wavelengths.

Upconversion lasers, emitting at wavelengths shorter than their excitation, potentially avoid shortcomings of above-band-gap excitation of solid-state media. It is therefore perhaps not too surprising that the first $\mathrm{cw}$ ultraviolet solid-state laser was a neodymium upconversion laser, ${ }^{5}$ which illustrates the utility of long-wavelength pumping. Other upconversion lasers have been operated recently in the visible spectral region on the basis of cooperative upconversion $^{6}$ and avalanche upconversion, ${ }^{7}$ and roomtemperature operation has been achieved in fibers ${ }^{8}$ and $\mathrm{BaYYb}_{0.99} \mathrm{Tm}_{0.01} \mathrm{~F}_{8}$ crystals. $^{9}$ Here we investigate a scheme for short-pulse generation with a visible Er: $\mathrm{LiYF}_{4}$ upconversion laser pumped in the midinfrared and emitting in the green spectral region. Continuous mode locking and $Q$ switching have been achieved for what is to our knowledge the first time with the use of intracavity amplitude modulation, indicating that upconversion lasers at blue or ultraviolet wavelengths offer an important approach to the direct generation of picosecond pulses in these spectral regions.

The cooperative upconversion laser on which this research is based attains threefold upconversion of pump-photon energy and $\mathrm{cw}$ emission in the green with $12 \%$ slope efficiency at temperatures as high as $95 \mathrm{~K}^{6}{ }^{6}$ In this device, inversion is due entirely to an energy-pooling process that involves three atoms, which is similar in nature to the operating principle of a monolithic Er: $\mathrm{CaF}_{2}$ trio laser that we described previously. ${ }^{10}$ By introducing a three-mirror, astigmatically compensated cavity with $\mathrm{Er}: \mathrm{LiYF}_{4}$ as the gain medium, we have been able to modulate losses in a fashion not possible in the original trio laser and find that this laser offers exceptional stability for mode-locking applications in comparison with earlier green erbium upconversion lasers. ${ }^{11}$

The experimental apparatus is shown in Fig. 1. The Brewster-oriented $\left(\theta_{B}=55.6^{\circ}\right)$ gain medium in the present experiment consisted of a 3-mm-thick crystal of $5 \% \mathrm{Er}: \mathrm{LiYF}_{4}$ with its optic axis parallel to the crystal surface, in the plane of incidence of the horizontally polarized pump field. This orientation permits gain extraction on both $\pi$ - and $\sigma$-polarized transitions. A tunable, $\mathrm{cw} \mathrm{NaCl}$ color-center laser provided resonant excitation of individual ${ }^{4} I_{13 / 2}$ Stark levels of $\mathrm{Er}^{3+}$ near $1.500 \mu \mathrm{m}$, and an interarm angle of $\theta=26.2^{\circ}$ compensated for astigmatism introduced by the two 5-cm-radius curved mirrors at the output wavelength of $544 \mathrm{~nm}$.

$Q$-switched operation was achieved by using a solid-state switch to control the amplitude of

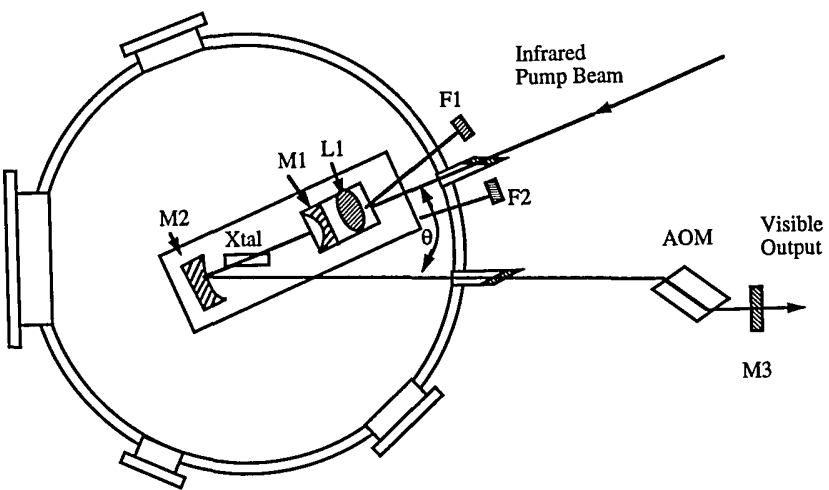

Fig. 1. Astigmatically corrected, three-mirror upconversion laser cavity. The Er: $\mathrm{LiYF}_{4}$ crystal (Xtal) is suspended on a cold finger at the center of the cavity within the vacuum chamber. A standing-wave, intracavity acousto-optic modulator (AOM) introduces variable loss at $119 \mathrm{MHz}$ near the output coupler, outside the Dewar. M's, mirrors; L's, lenses; F's, mechanical feedthroughs. 


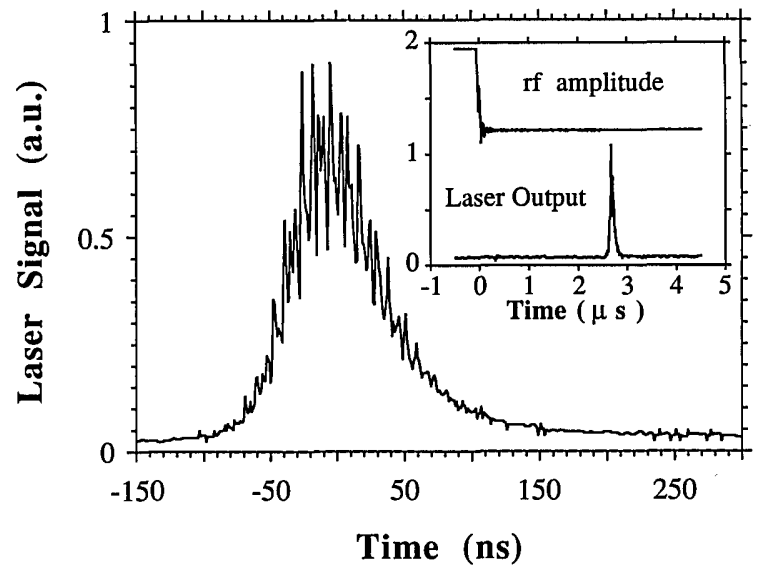

Fig. 2. Q-switched, upconversion laser output $(0.5440 \mu \mathrm{m})$ versus time at $9 \mathrm{~K}$. Inset: switching pulse sequence applied to the $\mathrm{rf}$ gate of the modulator driver and relative timing of output pulse.

$80-\mathrm{MHz}$ rf delivered to the driver amplifier of an antireflection-coated acousto-optic modulator. Square pulses with a repetition rate of $1 \mathrm{kHz}$ and an on/off duty cycle of $12 / 1$ furnished an energy storage cycle of $920 \mu \mathrm{s}$, which approaches the lifetime of the ${ }^{4} I_{13 / 2}$ state $(13.9 \mathrm{~ms}),{ }^{12}$ and a switch-out period of $80 \mu \mathrm{s}$. The cw pump laser was operated in a single transverse mode, with multiple longitudinal modes. Typically $Q$-switched pulses of $0.6-\mu \mathrm{J}$ energy and 50 -ns duration were obtained for a $\mathrm{cw}$ pumping power of $500 \mathrm{~mW}$. Peak output power was $9 \mathrm{~W}$, as shown in Fig. 2.

Mode-locked operation was achieved by insertion of a Brewster-angle, standing-wave modulator in the cavity near the output mirror and adjustment of the cavity length. The acoustic resonant frequency was $60.016 \pm(n \times 1.9675) \mathrm{MHz}$. A working frequency of $119.044 \mathrm{MHz}(n=30)$ was chosen to maintain a short, stable upconversion laser cavity. The output coupler was carefully translated parallel to the cavity axis until the cavity round-trip time matched the second-harmonic period (4.2 ns), at a cavity length of approximately $60 \mathrm{~cm}$. An Antel photodiode was mounted on the SD-24 sampling head of a $20-\mathrm{GHz}$ Tektronix 11802 digital oscilloscope to monitor the output waveform in real time. Results are shown in Fig. 3. Pulse formation was sensitive to cavitylength adjustments. The shortest pulses were initiated only within $\sim 50 \mu \mathrm{m}$ of the synchronous length (Fig. 4).

The output pulse train consisted of $\sim 200$-ps pulses spaced by the round-trip time of $4.2 \mathrm{~ns}$. Bandwidth limitations of the optical sampling and detector accounted for 50 and 45 ps (FWHM), respectively, hence the averaged traces furnished a direct upper limit on optical pulse width of $\sim 180$ ps after Gaussian deconvolution. Since an undetermined portion of this width was due to sampling jitter associated with the sine-wave signal used to trigger the scope, optical autocorrelation measurements would be useful to determine the pulse width more precisely. The inhomogeneous bandwidth of the upconversion laser transition is not known at present, hence we were unable to make a direct comparison between pulse and transition bandwidths. However, the observed pulse widths are consistent with inhomogeneous broadening in excess of $5 \mathrm{GHz}$, typical of rare-earth transitions in crystalline media at low temperatures, and no dependence on modulation depth was observed above the onset of mode locking with only $200-\mathrm{mW}$ rf input at $9 \mathrm{~K}$. Shorter modelocked upconversion laser pulses can undoubtedly be achieved with homogeneous mode locking in Er: $\mathrm{LiYF}_{4}$ at higher temperatures or in glass hosts. Upconversion fiber lasers should be particularly well suited to the direct generation of visible and ultraviolet pulses as short as a few picoseconds at room temperature.

Our mode locker was designed for use at $1.06 \mu \mathrm{m}$ and exhibited high insertion loss at visible wavelengths. The threshold for mode locking was $250 \mathrm{~mW}$, compared with $25 \mathrm{~mW}$ for cw operation. Similarly, a maximum average output of $2 \mathrm{~mW}$ was obtained in the mode-locked condition, compared with $20 \mathrm{~mW}$ in cw operation with the same $2 \%$ output coupler and $450 \mathrm{~mW}$ of incident pump power. Maximum operating temperature also dropped from $95 \mathrm{~K}$ to $15 \mathrm{~K}$ on insertion of the modulator. The performance can be expected to improve significantly by careful design of the modulator for the upconversion application.

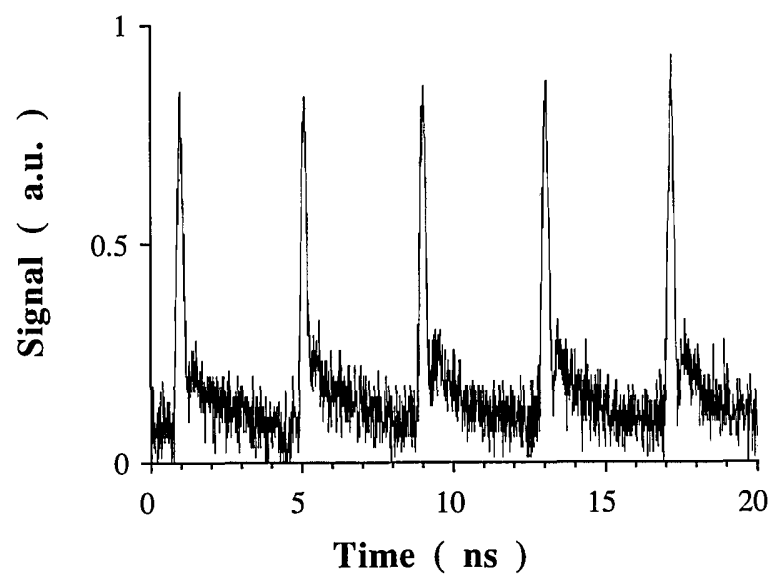

Fig. 3. Mode-locked output versus time at $0.5440 \mu \mathrm{m}$ for an infrared pump power at $1.5 \mu \mathrm{m}$ of $500-\mathrm{mW}$ power focused to a spot of radius $18 \mu \mathrm{m}$ in the gain medium.

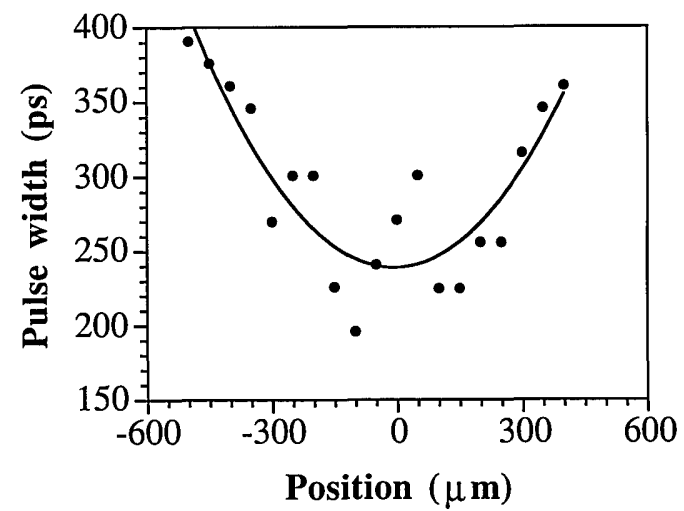

Fig. 4. Mode-locked pulse width versus position of the output coupler. The solid curve is the best fit of a quadratic regression. 
Excitation of any erbium absorption line in the range 1.45-1.55 $\mu \mathrm{m}$ resulted in laser output at $\lambda=$ $0.5440 \mu \mathrm{m}$ at $10 \mathrm{~K}$. This emission wavelength corresponds to the ${ }^{4} S_{3 / 2}(1)-{ }^{4} I_{15 / 2}(6)$ transition with mixed $\sigma$ and $\pi$ polarization as a result of Kramers degeneracy, terminating in the sixth ${ }^{4} I_{15 / 2}$ Stark level from the bottom of the manifold, $400 \mathrm{~cm}^{-1}$ above the true ground state. The spectrum of laser excitation reveals all seven ${ }^{4} I_{15 / 2}(1)-{ }^{4} I_{13 / 2}(n)$ Stark components, confirming that mode locking results from pumping into any ${ }^{4} I_{13 / 2}$ level at this temperature. At temperatures above $15 \mathrm{~K}$, the laser wavelength changed to $\lambda=0.5516 \mu \mathrm{m}^{6}$

An interesting advantage of the trio laser over earlier green erbium upconversion lasers for modelocking purposes is its operational stability in the $\mathrm{cw}$ mode. Continuous operation without spiking is obtained with the cooperative upconversion laser under all pumping conditions. This contrasts sharply with self-pulsing observed on the 551-nm transition in $\mathrm{Er}: \mathrm{LiYF}_{4}$ when alternate excitation methods are used, ${ }^{11}$ behavior that has been linked to excited-state absorption from the ${ }^{4} I_{13 / 2}$ level. ${ }^{13}$ Since the cooperative trio process relies on significant occupation of the ${ }^{4} I_{13 / 2}$ level, this difference in behavior cannot result from the absence of self-absorption. Rather, steady output must arise from inherently sluggish response of the cooperative upconversion mechanism to changes in intracavity photon density, compared with faster response times of multiphoton absorption mechanisms operative in other upconversion lasers. This appears to be essential in preventing uncontrolled, self-Q-switching at this wavelength in $\mathrm{LiYF}_{4}$.

In summary, we have demonstrated controlled $Q$ switching and active mode locking of a cooperative upconversion laser with an open cavity configuration. Pulses of less than 200-ps duration have been generated directly in the green spectral region by using cw pumping in the midinfrared. From the present results, and our earlier time-resolved spectroscopy, which revealed that excitation mechanisms other than cooperative upconversion contribute negligibly to the inversion of this trio laser, it may be concluded that efficient, cw mode-locked upconversion laser operation can be sustained at short wave- lengths by spontaneous trio interactions alone. Cooperative pumping apparently stabilizes the inversion against self- $Q$-switching, permits surprisingly efficient operation to temperatures as high as $95 \mathrm{~K}$, and should permit direct generation of $\mathrm{cw}$ modelocked picosecond pulses at much shorter wavelengths in this and other rare-earth solids.

The authors gratefully acknowledge sponsorship by the U.S. Air Force Office of Scientific Research, partial support by the National Science Foundation's Science and Technology Center for Ultrafast Optical Science, discussions with G. Mourou, and technical assistance from S. Williamson and M. Stock of the University of Michigan, T. Juhasz of the University of California, San Diego, L. Turi of the University of California, Irvine, and B. Couillaud of Coherent Inc.

\section{References}

1. P. M. W. French and J. R. Taylor, Opt. Lett. 13, 479 (1988).

2. D. E. Spence, P. N. Kean, and W. Sibbett, Opt. Lett. 16, 42 (1991).

3. V. Petrov, D. Georgiev, and U. Stamm, Appl. Phys. Lett. 60, 1550 (1992).

4. M. Haase, J. Qiu, J. DePuydt, and H. Cheng, Appl. Phys. Lett. 59, 1272 (1991).

5. R. M. Macfarlane, F. Tong, A. J. Silversmith, and W. Lenth, Appl. Phys. Lett. 52, 1300 (1988).

6. P. Xie and S. C. Rand, "Visible cooperative upconversion laser in Er:LiYF,", Opt. Lett. (to be published).

7. M. E. Koch, A. W. Kueny, and W. E. Case, Appl. Phys. Lett. 56, 1083 (1990).

8. J. Y. Allain, M. Monerie, and H. Poignant, Electron. Lett. 26, 262 (1990).

9. R. J. Thrash and L. F. Johnson, in Digest of Meeting on Compact Blue-Green Lasers (Optical Society of America, Washington, D.C., 1992), paper ThB3.

10. P. Xie and S. C. Rand, Appl. Phys. Lett. 57, 1182 (1990).

11. R. A. McFarlane, Appl. Phys. Lett. 54, 2301 (1989); T. Hebert, R. Wannemacher, W. Lenth, and R. M. Macfarlane, Appl. Phys. Lett. 57, 1727 (1990).

12. H. Chou, Ph.D. dissertation, MIT Technical Rep. No. 26 (Massachusetts Institute of Technology, Cambridge, Mass., 1989).

13. L. F. Johnson and H. J. Guggenheim, Appl. Phys. Lett. 20, 474 (1972). 